\title{
Non-classical methods of activation in the chemistry of heterocyclic compounds
}

Effective techniques of energy transfer into reaction systems are of key importance for advancing the art of chemical synthesis, along with the design of new catalytic systems or methods of physicochemical and spectral analysis. For example, one of the significant early innovations in chemical synthesis equipment was the invention of Bunsen burner, enabling precise control of the heat supplied to reaction mixtures. The range of methods available to synthetic chemists for the activation of chemical processes has significantly expanded since then and now includes photo- and electrochemical procedures, ultrasonication and microwave irradiation, mechanochemical processes, microreactor technologies, and many other methods, a few of which are still viewed as nonclassical.

This special issue of our journal is focused mainly on the use of microwave and ultrasonic methods in the chemistry of heterocyclic compounds, while also describing some aspects of mechanochemistry and the applications of flow reactors. These methods of activation have significantly influenced the creation and development of such current directions of chemistry as green chemistry, diversity-oriented synthesis, advancing medicinal and combinatorial chemistry, and many other valuable efforts.

While the physical mechanisms underlying the effects of microwave irradiation on chemical systems are still discussed in the literature, microwave activation is actively used in chemical synthesis, allowing not only to significantly shorten the reaction duration, but also to increase selectivity and, therefore, also the yields and purity of the target compounds. Furthermore, microwave techniques enable homogeneous heating of reaction mixtures within reactors, while avoiding local overheating and preventing side reactions of thermal degradation, allowing to work at supercritical temperatures for a wide range of solvents, and enabling precise control of chemical reaction parameters. The simple reaction procedures, shorter duration of synthesis, the flexibility in selecting solvents and reaction parameters, the reproducibility of the latter on analogous equipment at any laboratory, as well as many other factors make microwave irradiation a convenient tool in the arsenal of modern synthetic chemist.

Ultrasonication, on the other hand, is typically used for performing reactions at temperatures not higher than 50 $60^{\circ} \mathrm{C}$. While there is no direct interaction between ultrasound and vibrational or electronic energy levels of reactants, an indirect mechanism of action exists, based both on cavitation phenomena, as well as enhanced mass transfer in the reaction volume. The simple technical design, high effectiveness at room temperature, and

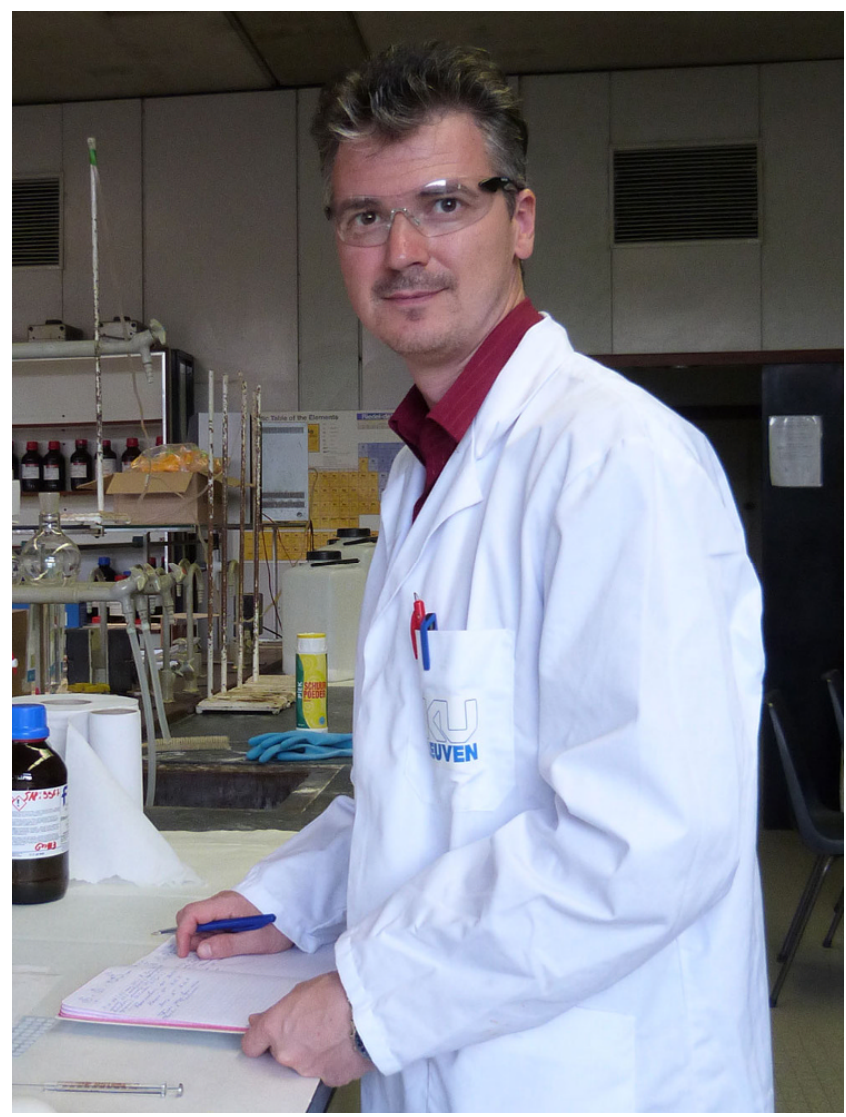

acceleration of processes by up to several tens of times give an important role to ultrasonic activation among other advanced methods of organic synthesis.

In the name of the editorial board, I express my gratitude to all authors who contributed to this thematic issue, as well as reviewers of the manuscripts, whose remarks and comments helped to improve the quality of articles and reviews offered here. It is hoped that the work of our team will allow the readers to better appreciate the convenience and effectiveness of non-classical methods of activation in solving a wide range of current chemistry problems, including the synthesis of heterocyclic compounds.

\section{Editor of the thematic issue Professor, Doctor of Chemical Sciences V. A. Chebanov, State Scientific Institution \\ "Institute for Single Crystals" of National Academy of Sciences}

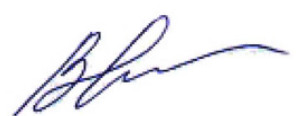
of Ukraine 